\title{
Exploring the natural chemiome to target interleukin-6 receptor (IL-6R) cytokines: an atomic scale investigation for novel rheumatoid arthritis drug discovery
}

\author{
Qiujian Chen ${ }^{1}$, Xiaohui Niu², Nana Li ${ }^{1 *}$ \\ ${ }^{1}$ College of Science, Huazhong Agricultural University, Wuhan, P.R. of China, ${ }^{2}$ College of Informatics, Huazhong Agricultural \\ University, Wuhan, P.R. of China
}

\begin{abstract}
Natural compounds are a gold mine for treating rheumatoid arthritis (RA). The etiology of this disease is linked to inflammation, where cytokines play an important role. Strategies have been drafted for targeting cytokines as a therapeutic option in patients with RA. Inhibiting cytokines with natural compounds has become a major focus for the development of drugs to treat RA. Here, a structure-based drug design approach was employed to identify novel leads to target the interleukin 6 receptor (IL-6R). A total of 48,531 compounds of natural origin were screened. Two of these compounds were shortlisted for molecular docking simulation and tested for inhibiting gp130 dimerization in human macrophages. The results show that Lead5 (CID5329098) significantly inhibited the release of gp130 in a dose-dependent manner, similar to the inhibitory effect of LMT-28 $(\mathrm{p}<0.005)$. This study provides an atomic scale outcome of a single natural compound that can be developed into a RA drug.
\end{abstract}

Keywords: Rheumatoid arthritis/treatment/natural compounds. Cytokines/therapeutic target. Docking. Natural compounds/study.

\section{INTRODUCTION}

Rheumatoid arthritis (RA) is a chronic inflammatory disease primarily associated with the joints that leads to cartilage and bone damage (Aletaha et al., 2010; Firestein, 2003). Inflammation is a part of the natural defense mechanism, but any anomaly can lead to various pathologies including RA (Khandpur et al., 2013; Lazzerini et al., 2014). Over the last decade, researchers have investigated the role of cytokines in the etiology of RA and have subsequently opted for cytokines as a therapeutic target (Burmester, Feist, Dörner, 2014; Siebert et al., 2015). Inhibiting cytokines has become a major focus in the development of new drugs to treat RA (Wu et al., 2016; Zhou et al., 2014).

The current therapeutic options in practice are various analgesics and disease-modifying anti-rheumatic drugs (DMARDs). DMARDs are further classified into conventional or synthetic DMARDs (c/sDMARDs)

\footnotetext{
*Correspondence: N. Li. College of Science, Huazhong Agricultural University. Wuhan 430070, P.R. of China. Tel/Fax: 0086-027-87282425. E-mail: nanali198@hotmail.com
}

and biological DMARDs (bDMARDs) (Al-Shakarchi, Gullick, Scott, 2013; Lambert, 2009). bDMARDs specifically target the cytokine network (Okuda, 2008) and are classified as anti-CD80/86 bDMRDs, anti-CD20 bDMRDs, anti-IL-1R bDMRDs, tumor necrosis factor (TNF)- $\alpha$ bDMRDs and anti-IL-6R bDMRDs (Hushaw et al., 2010; Kim et al., 2015). Tocilizumab (TCZ) is a monoclonal antibody sDMARD and the only approved anti-IL-6R drug (Nishimoto, Kishimoto, 2008), but it is associated with numerous side effects (Smolen et al., 2014). To overcome this difficulty, more and more research is being done on natural compounds (Smolen et al., 2007). Mining of natural products to discover natural leads against common diseases, and RA in particular, is a common practice (Amin et al., 2016; Chikan et al., 2013; Khanna et al., 2007).

In our study, we used the natural chemiome, as in interbioscreen (IBS) database of 48,531 compounds, known to be the world's largest collection of natural compounds, their derivatives, and mimetics. The database was used to target the crystallographic structures of the IL-6R extracellular domain (PDBID:1N26) (Varghese et al., 2002). In-silico techniques were used for an atomic 
scale investigation into possible IL-6R bDMARDs; virtual screening and molecular docking simulations were conducted to find a lead compound for in vitro analysis of its anti-inflammatory activity.

\section{MATERIAL AND METHODS}

\section{Preparation of the proteins and the natural chemiome dataset}

The atomic coordinates for the IL-6R structure to develop novel bDMRDs were taken from the Protein Databank (PDB ID: 1N26). The coordinates were energy minimized using the Swiss PDB viewer (SPDBv). The root mean square deviation was monitored using the GROMOS96 43B1 force field (Van Gunsteren, 1996). A total of 45,000 natural compounds from the IBS database were used to target the IL-6R extracellular domain.

\section{Virtual screening drug likeliness prediction}

The ArgusLab suite was used to virtually screen the 48,531 compounds (Mark, 2010). Less than 1\% (323) of the compounds were shortlisted based on their binding energy $(\Delta \mathrm{G})$ calculations. A value of $-8 \mathrm{Kcal} / \mathrm{mol}$ was set as the cut off to get the initial subset of compounds. The selected compounds were further limited by subjecting them to rules set by Lipinski (2004). The Lipinski rule of five (RO5) parameters gave us ten compounds for further analysis.

\section{Molecular docking analysis}

A structure-based drug designing method was used, and the AutoDock 4.2 tool was employed for the molecular docking study (Morris et al., 2009). This tool calculates energy values by classifying energies as internal energy and torsional free energy. Internal energy is the sum of desolvation energy, hydrogen bonding energy, van der Walls energy, and electrostatic energy. Lamarckian genetic algorithm (GA) default parameters were used to calculate $\Delta \mathrm{G}$ for each shortlisted compound. A grid box $(40 \times 40 \times 40$ $\mathrm{A}^{\circ}$ ) was built around the IL-6R extracellular domains. The energy values generated and the binding mode with IL-6R were used to limit the list to two compounds.

\section{Molecular visualization \& molecular docking analysis}

The two complexes were studied using the Pymol visualization tool (DeLano, 2002) and Discovery Studio (Studio, 2013).

\section{Cell culture}

THP-1 monocytes were procured from the American Type Culture Collection (Manassas, VA, USA) and cultured in RPMI (Sigma-Aldrich, St. Louis, MO, USA) supplemented with $10 \%$ fetal bovine serum (Gibco, Grand Island, NY, USA) and $100 \mathrm{U} / \mathrm{ml}$ pen-strep (Gibco). The cells were grown under standard culture conditions at $37^{\circ} \mathrm{C}$ and $5 \% \mathrm{CO}_{2}$ in a humidified incubator. Monocytes for macrophage differentiation were cultured with $100 \mathrm{ng} /$ $\mathrm{ml}$ macrophage colony stimulating factor (CSF). Stock solutions of $100 \mathrm{mM}$ of the compounds were prepared in RPMI and diluted to different concentrations. Cells were stimulated with $10 \mathrm{ng} / \mathrm{ml}$ lipopolysaccharide (LPS) (Sigma-Aldrich) to stimulate TNF- $\alpha$ production.

\section{Cell proliferation assay}

The MTT assay is a standard test to measure cell viability and is based on the conversion of MTT to formazan crystals by active mitochondrial dehydrogenases. Briefly, a cell suspension containing about $3 \times 10^{4}$ cells was plated into each well of a 96-well plate and allowed to attach for $24 \mathrm{~h}$. Lead 3 and Lead 5 prepared in DMEM were added to the wells at concentrations of 10, 20, 40, and 60,80 , and $100 \mu \mathrm{M}$ for $24 \mathrm{~h}$. After treatment, $100 \mu \mathrm{l}$ $(0.1 \mathrm{mg} / \mathrm{ml})$ of MTT (Sigma Aldrich) solution was added to each well (dissolved in PBS). After a $4 \mathrm{~h}$ incubation at $37{ }^{\circ} \mathrm{C}$ in the dark, the solution was removed and $100 \mu \mathrm{l}$ DMSO was added to solubilize the formazan. Absorbance was measured at $570 \mathrm{~nm}$ using an automated microplate reader (Biotek Instruments, Winooski, VT, USA). The results are depicted as percentages relative to the controls. The percentage proliferation inhibition rate was calculated as $=(1-$ ODsample/ODcontrol $) \times 100 \%$.

\section{gp130 Enzyme-linked immunosorbent assay (ELISA)}

The supernatant was harvested following Lead 3 and Lead 5 stimulation of human macrophages with LPS. gp130 concentrations (BD Pharmingen, San Diego, CA, USA) were determined by ELISA, following the manufacturer's instructions. Absorbance was read at 450 $\mathrm{nM}$ on an ELISA plate reader (Biotek) using the in-built software program.

\section{Statistical analysis}

The statistical analysis was carried out by one-way analysis of variance, and p-values of 0.01-0.001 were 
considered significant. Data are expressed as mean $\pm \mathrm{SD}$ of three independent experiments.

\section{RESULTS AND DISCUSSION}

\section{Interleukin-6/JAK/STAT pathway inhibition by IBS database}

Chemiomes are comprised of natural compounds, their derivatives, and mimetics that inhibit the IL-6/ JAK/STAT pathway by inhibiting the IL-6R (Figure 1). IL-6R in complex with IL-6 results in homodimerization of gp130 and signal transduction through the JAK/ STAT pathway. Thus, blocking formation of the IL-6 and IL-6R complex by targeting the IL-6R extracellular domains resulted in a novel IL-6R inhibitor by virtual screening, drug-likeliness, docking and molecular dynamics simulation studies. Figure 2 depicts this methodology. Virtual screening helped limit the number of compounds from the 48,531 natural products to 323 using a limiting bias of $\Delta \mathrm{G}$ of $-8 \mathrm{Kcal} / \mathrm{mol}$. Figure 3 shows the X-ray crystallographic structure of one of the natural compounds with IL-6R.

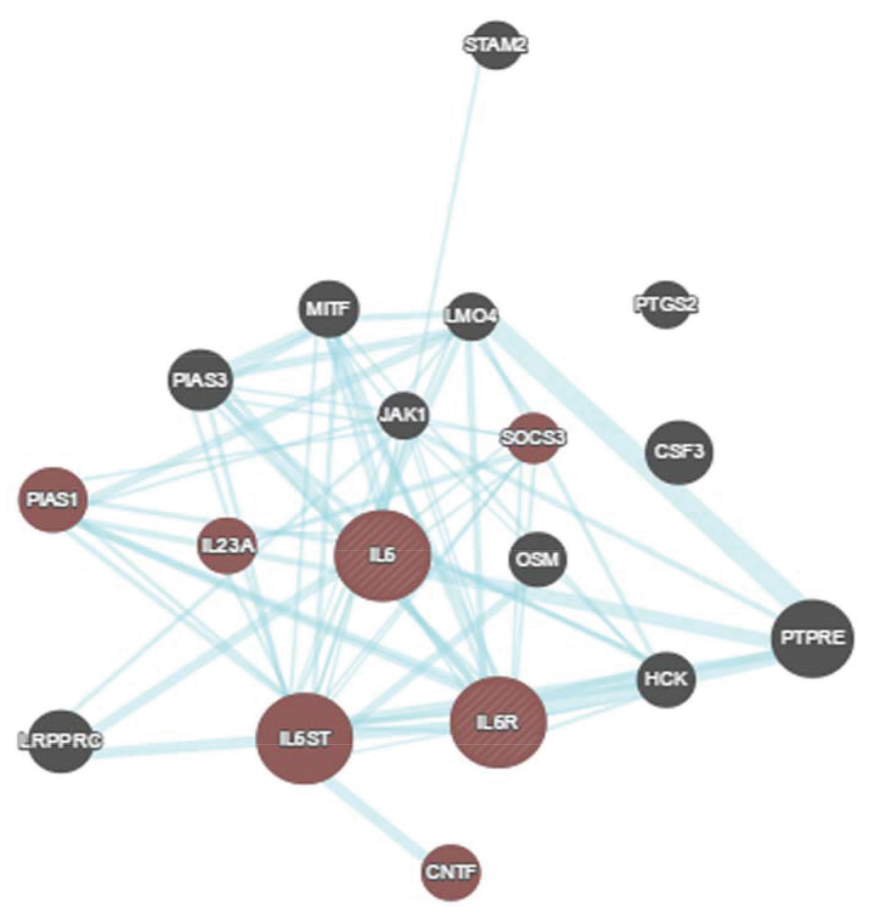

FIGURE 1 - Protein sub networks of IL-6R with other important proteins involved in arthritis.

\section{Drug likeliness feature of the top compounds}

To limit the focus on compounds that could be promising for further development, we checked each

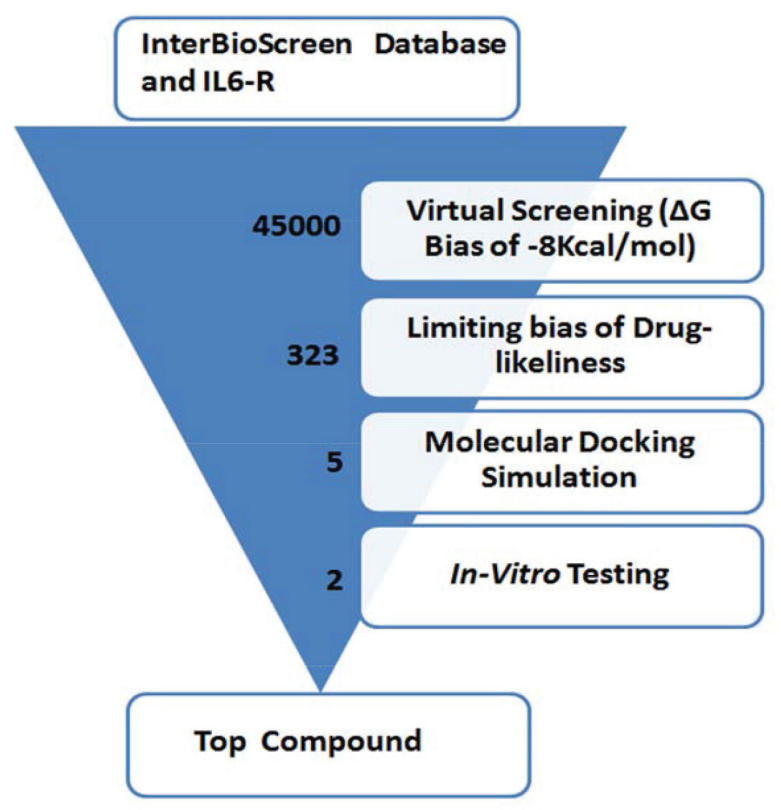

FIGURE 2 - Method used for screening of natural compounds against IL-6R.

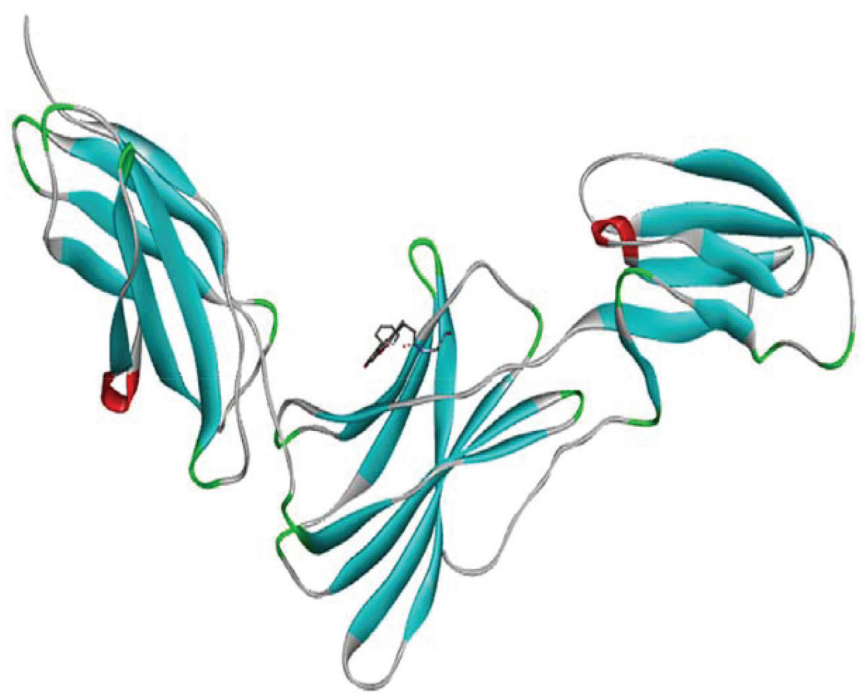

FIGURE 3 - The original crystallographic structure of IL-6R used for the study.

compound for drug-likeliness. Drug-likeliness of the shortlisted compounds was defined by mutagenic and carcinogenic properties, RO5, and total polar surface area. The RO5 properties included the number of hydrogen bond donors (HBD), number of hydrogen bond acceptors (HBA), molecular weight (MW), and the octanol/water partition coefficient $(\log \mathrm{P})$. The permissible range is $\mathrm{HBD} \leq 5, \mathrm{HBA} \leq 10, \mathrm{MW} \leq 500$ Dalton and $\operatorname{clog} \mathrm{p} \leq 5$. Table I shows the drug-likeliness properties of the top five compounds. The drug-likeliness values of our top compounds are values expected of typical drugs. 
TABLE I - Details of the top five compounds

\begin{tabular}{lccccc}
\hline Name & Canonical SMILES & MW & HBD & HBA & tpsa \\
\hline Lead 1 & $\mathrm{C} 1=\mathrm{CC}=\mathrm{C} 2 \mathrm{C}(=\mathrm{C} 1) \mathrm{C}(=\mathrm{CN} 2) \mathrm{CCC} 3=\mathrm{CC}=\mathrm{NC}=\mathrm{C} 3$ & 222.291 & 1 & 1 & 28.7 \\
Lead 2 & $\mathrm{CNC}(=\mathrm{O}) \mathrm{C} 1=\mathrm{NC}=\mathrm{CC}(=\mathrm{C} 1) \mathrm{OC} 2=\mathrm{CC}=\mathrm{C}(\mathrm{C}=\mathrm{C} 2) \mathrm{NC}(=\mathrm{O})$ & 464.829 & 3 & 7 & 92.4 \\
$\mathrm{NC} 3=\mathrm{CC}(=\mathrm{C}(\mathrm{C}=\mathrm{C} 3) \mathrm{Cl}) \mathrm{C}$ & & & & 74.7 \\
Lead 3 & $\mathrm{COCCOC} 1=\mathrm{C}(\mathrm{C}=\mathrm{C} 2 \mathrm{C}(=\mathrm{C} 1) \mathrm{C}(=\mathrm{NC}=\mathrm{N} 2) \mathrm{NC} 3=\mathrm{CC}=\mathrm{CC}(=\mathrm{C} 3)$ & 393.433 & 1 & 7 & \\
& $\mathrm{CC}) \mathrm{OCCOC}$ & & & 44.9 \\
Lead 4 & $\mathrm{CC} 1=\mathrm{CC}(=\mathrm{C}(\mathrm{N} 1) \mathrm{C}=\mathrm{C} 2 \mathrm{C} 3=\mathrm{CC}=\mathrm{CC}=\mathrm{C} 3 \mathrm{NC} 2=\mathrm{O}) \mathrm{C}$ & 238.290 & 2 & 1 & 58.21 \\
Lead 5 & $\mathrm{CC}(=\mathrm{C}) \mathrm{COC} 1 \mathrm{NC}(\mathrm{C} 2 \mathrm{C}(\mathrm{C} 1 \mathrm{C} \# \mathrm{~N}) \mathrm{C}[\mathrm{C}](\mathrm{OC} 2)(\mathrm{C}) \mathrm{C}) \mathrm{N} 3 \mathrm{CCCC} 3$ & 242.426 & 1 & 5 & \\
\hline
\end{tabular}

\section{Intricate atomic scale details of the interactions in} the top five lead compounds

The AutoDock tool was used for the molecular docking simulations, and the top binding poses based on $\Delta \mathrm{G}$ were taken for further analysis. Each binding pose was studied using Discovery Studio, and the default parameters were used to calculate all possible interactions. The results generated are shown in Table II, where the binding energy, binding pocket, and number of hydrogen bonds formed are listed. The lead1-IL-6R complex had a binding energy of $-4.43 \mathrm{kcal} / \mathrm{mol}$ and their interaction is shown in Figure 4, where lead1 formed three conventional hydrogen bonds with LYS154, PHE155, and GLU114 in the IL-6R GTP binding sites. The binding pocket of lead1 was comprised of the following amino acids LYS154, PHE155, GLY116, SER152, TRP115, GLN99, SER101, CYS113, GLU114, VAL112, SER156, LEU100, and CYS157. The binding energy was third least among five, and the number of interactions was the least among all shortlisted compounds.

Lead 2 formed six conventional hydrogen bond interactions with the IL-6R binding pocket. The lead 2 binding pocket was comprised of 21 amino acids: LEU129, VAL112, GLU114, LEU100, CYS102, CYS113, SER101, TRP115, PHE103, GLY116, GLN99, SER149, TYR148, GLN150, GLU151, GLN153, SER152, LYS154, PHE155, MET173, and CYS157. Lead 2 had a $\Delta \mathrm{G}$ of $-2.31 \mathrm{kcal} / \mathrm{mol}$, which was the lowest reported among the shortlisted top five compounds. The binding pocket of the lead 3 (CID176870) molecule with IL-6R was comprised of the following amino acids viz. CYS157, GLN147, SER156, GLY116, SER152, PHE155, LYS154, SER149, TYR148, PRO117, GLU151, GLN153, TRP115, SER101, GLU114, CYS102, VAL112, CYS113, LEU100, GLN99, and PHE103. Of them, lead3 formed hydrogen bonds with CYS102, GLU114, SER101, TRP115, SER156, and PHE155, and the interactions were maximum among the five lead compounds.

TABLE II - Auto Dock analysis of top five lead natural products

\begin{tabular}{|c|c|c|c|}
\hline Name & $\begin{array}{c}\Delta \mathrm{G} \\
\mathrm{Kcal} / \mathrm{mol}\end{array}$ & Ligand binding pocket & H-bonds \\
\hline Lead 1 & -4.43 & $\begin{array}{l}\text { LYS154, PHE155, GLY116, SER152, TRP115, GLN99, SER101, CYS113, GLU114, } \\
\text { VAL112, SER156, LEU100, CYS157 }\end{array}$ & Three \\
\hline Lead 2 & -2.31 & $\begin{array}{l}\text { LEU129, VAL112, GLU114, LEU100, CYS102, CYS113, SER101, TRP115, PHE103, } \\
\text { GLY116, GLN99, SER149, TYR148, GLN150, GLU151, GLN153, SER152, LYS154, } \\
\text { PHE155, MET173, CYS157 }\end{array}$ & Six \\
\hline Lead 3 & -7.21 & $\begin{array}{l}\text { CYS157, GLN147, SER156, GLY116, SER152, PHE155, LYS154, SER149, TYR148, } \\
\text { PRO117, GLU151, GLN153, TRP115, SER101, GLU114, CYS102, VAL112, CYS113, } \\
\text { LEU100, GLN99, PHE103 }\end{array}$ & Ten \\
\hline Lead 4 & -3.21 & $\begin{array}{l}\text { LEU100, GLN187, CYS113, CS102, GLU114, TRP115, VAL112, PHE155, SER152, } \\
\text { GLN153, GLY116, SER101, SER156, LYS154, PRO117, SER149, TYR148 }\end{array}$ & Four \\
\hline
\end{tabular}



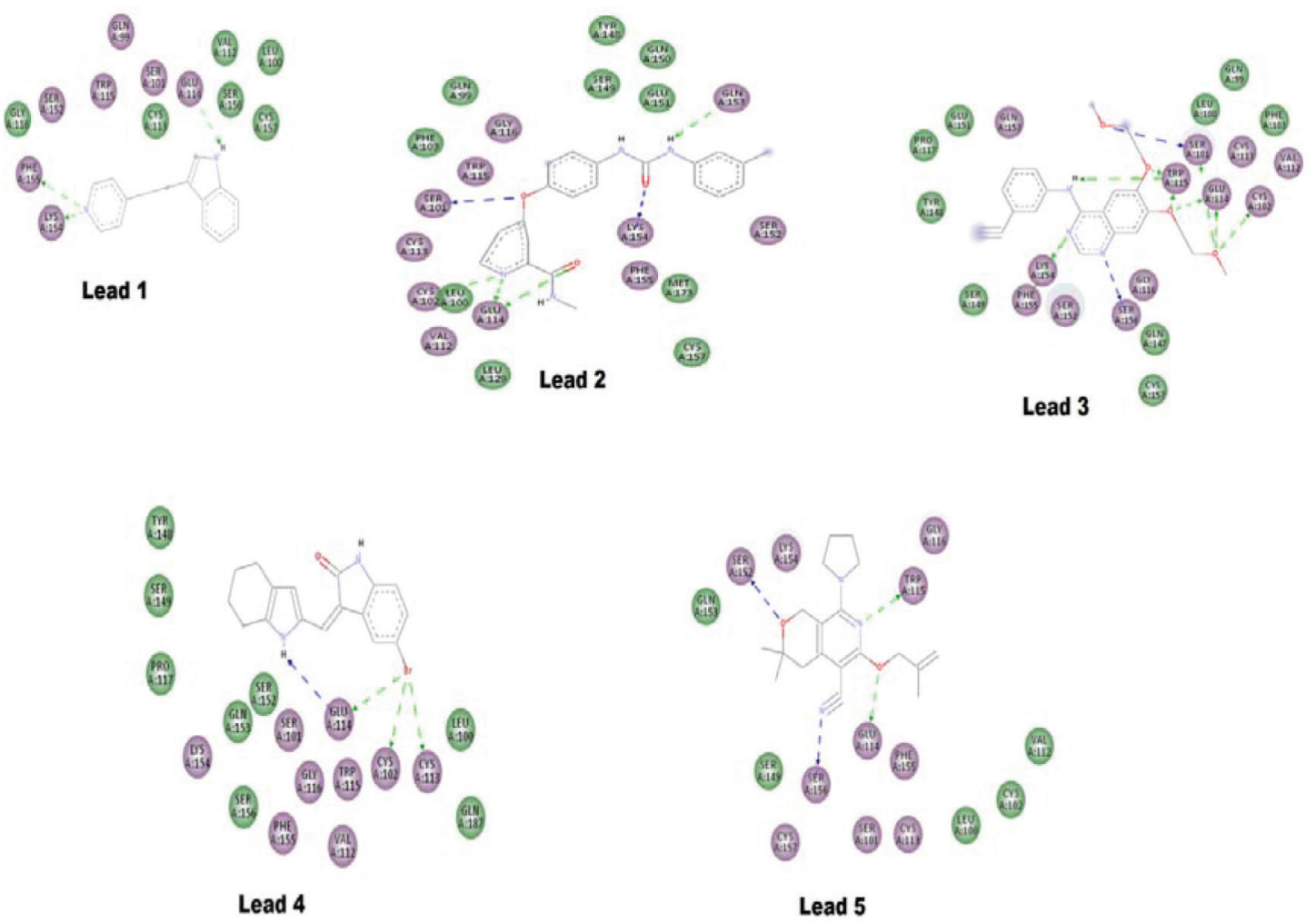

FIGURE 4 - Two dimensional representations of the top five compounds showing the physical interaction with the binding site of IL-6R.

Based on $\Delta \mathrm{G}$ and the number of interactions, lead4 was the least ranked among the top five compounds. Its binding pocket was comprised of 17 amino acids; LEU100, GLN187, CYS113, CS102, GLU114, TRP115, VAL112, PHE155, SER152, GLN153, GLY116, SER101, SER156, LYS154, PRO117, SER149, and TYR148. Lead4 had a $\Delta \mathrm{G}$ of $-3.21 \mathrm{Kcal} / \mathrm{mol}$ and had four hydrogen bond interactions. The last lead compound, lead5 (CID5329098) had a $\Delta \mathrm{G}$ of $-6.38 \mathrm{Kcal} / \mathrm{mol}$. Lead5 formed four interactions with the IL-6R binding pocket. The lead5 binding pocket was comprised of VAL112, CYS102, LEU100, CYS113, PHE155, SER101, GLU114, SER156, CYS157, SER149, SER152, LYS154, TRP115, and GLY116.

\section{Inhibiting dimerization of gp130 by human macrophages}

gp130 is a key factor in a variety of inflammatory diseases. We evaluated its expression in the culture supernatants of human macrophages (which constitute the major cytokine-producing cells in highly relevant inflammatory disorders) treated with non-cytotoxic doses of Lead3 (CID176870) and Lead5 (CID5329098), considering the role of IL- 6 and IL-6R regulating gp130 dimerization, to determine the suitable dosage of Lead 3 and Lead 5 compounds that result in less cytotoxicity. Macrophages (obtained with treatment of THP-1 monocytes with CSF) were treated with different concentrations of these compounds $(10-200 \mu \mathrm{M})$ for $24 \mathrm{~h}$, and viability was measured by the MTT assay. The results demonstrated that treatment with concentrations such as $10-100 \mu \mathrm{M}$ exhibited the least cytotoxic effects. To test the effect of these lead compounds, macrophages were treated with the indicated concentrations of 0.001-100 $\mu \mathrm{M}$ of these two compounds with LMT-28, a selective inhibitor of IL-6R. Treating LPS stimulated macrophages with Lead5 (CID5329098) significantly inhibited release of gp130 in a dose dependent manner; similar to the inhibitory effect of LMT-28 (Figure 5). Thus, these data suggest a role inhibiting IL-6R that, in turn, downregulates gp130 and, hence, could be used a therapeutic agent to target RA.

\section{CONCLUSION}

Computer aided drug design was used to explore the natural chemiome for treating RA. The compounds have long has been described as a gold mine for arthritis treatment and studies have explored their ability to inhibit 


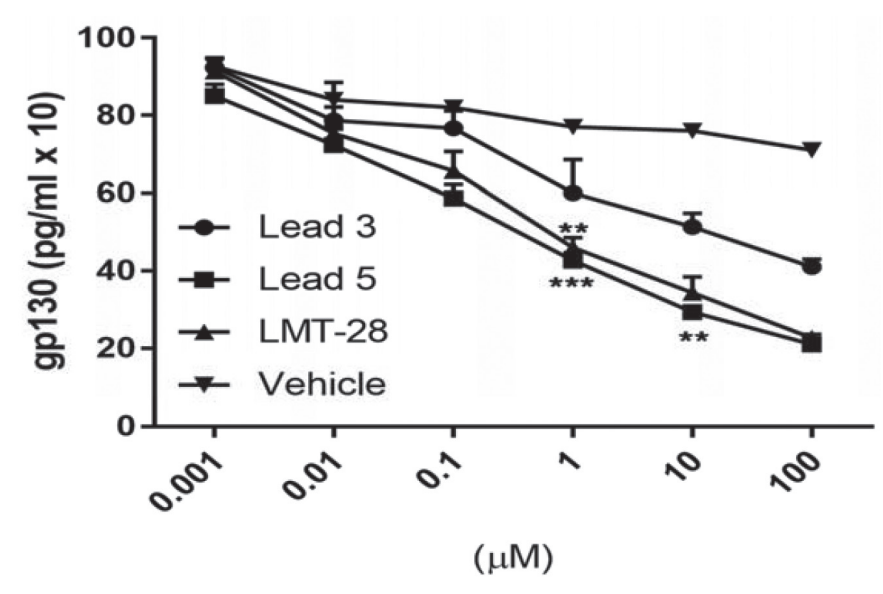

FIGURE 5 - Effect of top two lead compounds on gp130 dimerization in macrophages. Cells treated with indicated concentrations of these two compounds LMT-28 or left untreated. The culture supernatants were then removed and assayed for gp130 by ELISA. Data represented as mean \pm SD of results obtained from at least three independent experiments. $* * \mathrm{p}<0.01 ; * * * \mathrm{p}<0.001$ compared to control.

cytokines. Natural compounds have long been reported to have anti-rheumatoid effects, and this has given us an opportunity to look into them as IL-6R inhibitors. The molecular docking simulations studies revealed potential lead compounds, and some of these compounds are showing great potential as drugs for treatment of RA in preliminary in vitro investigations.

\section{REFERENCES}

Al-Shakarchi I, Gullick NJ, Scott DL. Current perspectives on tocilizumab for the treatment of rheumatoid arthritis: a review. Patient Prefer Adherence. 2013;4(7):7653-666.

Aletaha D, Neogi T, Silman AJ, Funovits J, Felson DT, Bingham CO, et al. 2010 rheumatoid arthritis classification criteria: an American College of Rheumatology/European League Against Rheumatism collaborative initiative. Arthritis Rheum. 2010;62(9):2569-2581.

Amin A, Chikan NA, Mokhdomi TA, Bukhari S, Koul AM, Shah BA, et al. Irigenin, a novel lead from Western Himalayan chemiome inhibits Fibronectin-Extra Domain A induced metastasis in Lung cancer cells. Sci Rep. 2016. (in press).

Burmester GR, Feist E, Dörner T. Emerging cell and cytokine targets in rheumatoid arthritis. Nat Rev Rheumatol. 2014,10(2):77-88.
Chikan NA, Bhavaniprasad V, Anbarasu K, Shabir N \& Patel TN. From natural products to drugs for epimutation computeraided drug design. Appl Biochem Biotechnol. 2013;170(1):164175.

DeLano WL. The PyMOL molecular graphics system. 2002.

Firestein GS. Evolving concepts of rheumatoid arthritis. Nature. 2003;423(6937):356-361.

Hushaw LL, Sawaqed R, Sweis G, Reigle J, Gopal A, Brandt $\mathrm{D}$, et al. Critical appraisal of tocilizumab in the treatment of moderate to severe rheumatoid arthritis. Ther Clin Risk Manag. 2010;15(6):143-152.

Khandpur R, Carmona-Rivera C, Vivekanandan-Giri A, Gizinski A, Yalavarthi S, Knight JS, et al. NETs are a source of citrullinated autoantigens and stimulate inflammatory responses in rheumatoid arthritis. Sci Transl Med. 2013;5(178):178ra140178 ra140.

Khanna D, Sethi G, Ahn KS, Pandey MK, Kunnumakkara $\mathrm{AB}$, Sung B, et al. Natural products as a gold mine for arthritis treatment. Curr Opin Pharmacol. 2007;7(3):344-351.

Kim GW, Lee NR, Pi RH, Lim YS, Lee YM, Lee JM, et al. IL-6 inhibitors for treatment of rheumatoid arthritis: past, present, and future. Arch Pharm Res. 2015;38(5):575-584.

Lambert DG. Disease-modifying anti-rheumatic drugs. Anaesth Intens Care. 2009;10(1):35-37.

Lazzerini PE, Capecchi PL, Acampa M, Galeazzi M, LaghiPasini F. Arrhythmic risk in rheumatoid arthritis: the driving role of systemic inflammation. Autoimmun Rev. 2014;13(9):936944.

Lipinski CA. Lead-and drug-like compounds: the rule-of-five revolution. Drug Discov Today Technol. 2004;1(4):337-341.

Mark A. Argus Lab 4.0.1. Thompson, Planaria Software LLC. 2010.

Morris GM, Huey R, Lindstrom W, Sanner MF, Belew RK, Goodsell DS, Olson AJ. AutoDock4 and AutoDockTools4: Automated docking with selective receptor flexibility. J Comput Chem. 2009;30(16):2785-2791.

Nishimoto N, Kishimoto T. Humanized antihuman IL-6 receptor antibody, tocilizumab. Handb Exp Pharmacol. 2008;181:151-60. 
Okuda Y. Review of tocilizumab in the treatment of rheumatoid arthritis. Biologics. 2008;2(1):75-82.

Siebert S, Tsoukas A, Robertson J, McInnes I. Cytokines as therapeutic targets in rheumatoid arthritis and other inflammatory diseases. Pharmacol Rev. 2015;67(2):280-309.

Smolen JS, Aletaha D, Koeller M, Weisman MH, Emery P. New therapies for treatment of rheumatoid arthritis. The Lancet. 2007;370(9602):1861-1874.

Smolen JS, van der Heijde D, Machold KP, Aletaha D, Landewé R. Proposal for a new nomenclature of disease-modifying antirheumatic drugs. Ann Rheumatic Dis. 2014;73(1):3-5.

Studio D. Accelrys Inc, San Diego, CA, USA. 2013.

Van Gunsteren WF. Biomolecular simulation: the GROMOS96 manual and user guide. Groningen: Biomos; Zürich: Vdf, Hochschulverlag AG an der ETH; 1996. 1044 p.
Varghese J, Moritz RL, Lou MZ, Van Donkelaar A, Ji H, Ivancic N, et al. Structure of the extracellular domains of the human interleukin-6 receptor $\alpha$-chain. Proc Natl Acad Sci USA. 2002;99(25):15959-15964.

Wu J, Qu Y, Deng J-X, Liang W-Y, Jiang Z-L, Lai R, et al. Resveratrol inhibition of TNF- $\alpha$ and IL-1 for treatment of rheumatoid arthritis: from In-Silico to In-vitro elucidation. Int J Clin Exp Med. 2016;9(2):745-752.

Zhou W, Cai J-F, Yuan F, Ma M, Yin F. In silico targeting of interleukin- 6 by natural compounds. Bangladesh J Pharma. 2014;9(3):371-376.

Received for publication on $04^{\text {th }}$ May 2017 Accepted for publication on $09^{\text {th }}$ June 2017 